TRANSACTIONS OF THE

AMERICAN MATHEMATICAL SOCIETY

Volume 358, Number 8, Pages 3305-3328

S 0002-9947(05)03964-4

Article electronically published on December 20, 2005

\title{
ON DECOMPOSITIONS IN HOMOTOPY THEORY
}

\author{
BRAYTON GRAY
}

\begin{abstract}
We first describe Krull-Schmidt theorems decomposing $H$ spaces and simply-connected co- $H$ spaces into atomic factors in the category of pointed nilpotent $p$-complete spaces of finite type. We use this to construct a 1-1 correspondence between homotopy types of atomic $H$ spaces and homotopy types of atomic co- $H$ spaces, and construct a split fibration which connects them and illuminates the decomposition. Various properties of these constructions are analyzed.
\end{abstract}

The Krull-Schmidt property first arose in the theory of $R$-modules, and when valid, it states that each object decomposes in a unique way into a sum of indecomposable objects of the same type. Numerous examples of decomposing the loop space on a co- $H$ space can be found in the literature ([Hi], [M], CM], AG], G1], G2]). Typically what happens is that the loop space of an atomic co- $H$ space is a product of various factors, and the least connected factor is an $H$ space of special interest, while the other factors, in some sense, represent noise. The first general Krull-Schmidt type theorem in homotopy theory was proved by Wilkerson [W] for the $p$-localization of simply-connected finite complexes which are either $H$ spaces or co- $H$ spaces. Various stable versions appear in $[\mathrm{F}$, $\mathrm{Ma}$ and $[\mathrm{H}]$.

We will eliminate the finite complex assumption at the expense of retreating to the category $\mathcal{C}_{p}^{\wedge}$ of pointed connected nilpotent $p$-complete spaces with $H_{i}(X ; \mathbb{Z} / p)$ finitely generated for each $i$. Accordingly, we restrict ourselves to this category in the sequel. All colimit constructions will be completed without further notice. In particular, co- $H$ spaces will be defined in terms of the coproduct (which is the completion of the one point union), suspensions will be completed and loop spaces will only be considered when the underlying space is simply connected.

In section 1 we will exploit the strengthened notion of atomicity in this category developed by Adams and Kuhn [AK], and prove a Krull-Scmidt theorem (Theorem A below).

Theorem A. Each $H$ space in $\mathcal{C}_{p}^{\wedge}$ is homotopy equivalent to the weak direct product of atomic $H$ spaces unique up to order. Each simply-connected co-H space in $\mathcal{C}_{p}^{\wedge}$ is homotopy equivalent to the coproduct of atomic complete co-H spaces unique up to order.

In section 2 we give a general correspondence between retracts of $n$-fold suspensions and retracts of $n$-fold loop spaces (2.2). In particular, for $n=1$ we get the following theorem.

Received by the editors February 5, 2004.

2000 Mathematics Subject Classification. Primary 55P35; Secondary 55P30, 55P45.

(C)2005 American Mathematical Society 
Theorem B. There is a 1-1 correspondence between homotopy types of atomic $H$ spaces $T$ in $\mathcal{C}_{p}^{\wedge}$ and homotopy types of 1 -connected atomic co-H spaces $G$ in $\mathcal{C}_{p}^{\wedge} \mathbb{1}$

We call such a pair $(G, T)$ a corresponding pair. In this corrrespondence, $T$ is a retract of $\Omega G$ and $G$ is a retract of $S T$ :

$$
\begin{array}{ll}
G \stackrel{f}{\longrightarrow} S T \stackrel{g^{\prime}}{\longrightarrow} G, & g^{\prime} f \sim 1, \\
T \stackrel{h}{\longrightarrow} \Omega G \stackrel{h}{\longrightarrow} T, & h g \sim 1 .
\end{array}
$$

In fact, in (2.5) it is possible to choose $g$ and $g^{\prime}$ so that they are adjoint. We will call the maps $\left(f, g, g^{\prime}, h\right)$ structure maps for the corresponding pair $(G, T)$. A choice of structure maps determines an $H$ space structure on $T$ and a co- $H$ space structure on $G$ via the compositions:

$$
\begin{aligned}
& m: T \times T \stackrel{g \times g}{\longrightarrow} \Omega G \times \Omega G \longrightarrow \Omega G \stackrel{h}{\longrightarrow} T, \\
& n: G \stackrel{f}{\longrightarrow} S T \longrightarrow S T \vee S T \stackrel{g^{\prime} \vee g^{\prime}}{\longrightarrow} G \vee G .
\end{aligned}
$$

A surprising amount of the structure of known examples is found in the general theory. In particular, given structure maps $\left(f, g^{\prime}, h\right)$ there is a fibration

$$
T \stackrel{i}{\longrightarrow} R \stackrel{\pi}{\longrightarrow} G
$$

with $i$ null homotopic. This leads to Theorem 3.2 and Corollary 3.12 which we summarize as

Theorem C. Suppose $T$ is an atomic $H$ space and $G$ is the corresponding atomic complete co-H space. Then there is a homotopy equivalence

$$
\Omega G \simeq T \times \Omega R,
$$

where $R$ is a retract of the completed join $T * T$ and hence a complete co-H space. Furthermore $R=\bigvee R_{\alpha}$ with each $R_{\alpha}$ atomic and

a) if $G=S X, R_{\alpha}$ is a retract of $S X^{(i)}$ for some $i \geq 2$,

b) if $G$ is homotopy co-associative, $S^{j-1} R_{\alpha}$ is a retract of $G^{(j)}$ for some $j \geq 2$,

c) if $G=S X$ and $G \wedge G \simeq \bigvee_{\alpha \in A} S^{n_{\alpha}} G, R \simeq G \wedge W$, where $W$ is a wedge of spheres and

$$
P_{W}=P_{T}-\frac{t\left(P_{T}-1\right)}{P_{G}-1}+1,
$$

where $P_{X}$ is the Poincaré series for $X$.

This result should be compared with the results of Selick and Wu [SW]. They have a similar decomposition in the case when $G$ is the double suspension of a $p$ torsion space:

$$
\Omega S^{2} X=A \times B,
$$

where $X$ is contained in $A$. $A$ however is much larger than $T$. In fact, $T$ is a retract of $A$.

\footnotetext{
${ }^{1}$ Theorems $\mathrm{A}$ and $\mathrm{B}$ together imply that homotopy types of quasi- $H$ spaces in $\mathcal{C}_{p}^{\wedge}$ are in 1-1 correspondence with homotopy types of simply-connected quasi-co- $H$ spaces in $\mathcal{C}_{p}^{\wedge}$. However we will not consider this correspondence
} 
In section 3 we construct the fibration which controls the splitting. Section 4 deals with the possibility of dualizing the material in section 3 and gives a counterexample to a conjecture of Ganea. Section 5 discusses naturality, and section 6 constructs a refinement in the case that $T$ has a homotopy associative multiplication. In section 7 we discuss criteria which are needed for the spece $T$ to have a homotopy associative $H$ space structure. Finally, in the Appendix, we collect some results of a general nature regarding fibrations over suspensions.

1.

Homology and cohomology groups will be with $\mathbb{Z} / p$ coefficients unless otherwise noted. We will work in the category $\mathcal{C}_{p}^{\wedge}$ of pointed connected nilpotent $p$-complete spaces of the homotopy type of a $C W$ complex $X$, with $H_{i}(X)$ finitely generated for each $i$. We need to arrange our definitions so that $p$-completion preserves the usual operations of homotopy theory. All spaces we consider will either be simply connected or admit $H$ space structures, so they will be $\mathbb{Z} / p$ good in the sense of $[\mathrm{BK}]$. All fibrations we consider will have a simply-connected base, so $p$-completion preserves fibrations. The $p$-completion of a co- $H$ space is often not a co- $H$ space. However, the category of $p$-complete spaces does have a co-productthe $p$-completion of the one point union:

$$
(X \vee Y)_{p}^{\wedge}
$$

and we will write $X \vee Y$ for this co-product. With this in mind, we define a co- $H$ space in $\mathcal{C}_{p}^{\wedge}$ to be a space $G$ together with a map

$$
\nu: G \longrightarrow G \vee G
$$

such that the composition with the natural map $G \vee G \longrightarrow G \times G$ is homotopic to the diagonal. Thus the $p$-completion of a co- $H$ space is a co- $H$ space in this sense. If $X$ is simply connected, we will consider $\Omega X$ and $(\Omega X)_{p}^{\wedge}=\Omega\left(X_{p}^{\wedge}\right)$. The functor $\Omega$ has a left adjoint given by the completion of the suspension, and we write $S X$ for the space $(S X)_{p}^{\wedge}$. Then $S X$ is a complete co- $H$ space. Similarly we write $X \wedge Y$ and $X * Y$ for the $p$ completions of the smash and join of two spaces. Then all of these operations commute with $p$-completion.

Adams and Kuhn discuss self maps of such spaces [AK. They show a close relationship between irreducibility of such spaces (having no nontrivial retracts) and atomicity. We summarize the results of $[\mathrm{AK}$ that we will need.

Definition 1.1. A based map $f: X \longrightarrow X$ is called topologically nilpotent if the sequence $\left\{f^{n}\right\}$ conveys to the constant map in the profinite topology on $[X, X]$.

In particular, if $\pi_{k}(X)$ is finite for each $k$, this is equivalent to the statement that for all $k$ there is an $n$ such that $f^{n}$ is null homotopic when restricted to the $k$-skeleton of $X$. On the other hand, if for some $k>0 f^{*}$ induces an isomorphism $H^{k}(X) \stackrel{f^{*}}{\longrightarrow} H^{k}(X) \neq 0$ or $f_{*}$ induces an isomorphism $f_{*}: \pi_{k}(X) \longrightarrow \pi_{*}(X)$, then $f$ is not topologically nilpotent.

Definition 1.2. A space is atomic iff every self map is either an equivalence or is topologically nilpotent.

This seems stronger than the usual definition $[\mathrm{CM}]$. The fact that it is not (in the case of $p$-complete spaces of finite type) follows from the next theorem. 
Theorem 1.3 (Adams and Kuhn). If $X$ is not atomic, there is a nontrivial idempotent $e: X \longrightarrow X$; i.e., $e^{2}=e, e \neq *, e$ not an equivalence.

As an immediate corollary we have a generalization of a result of Wilkerson W. in the $p$-complete case.

Corollary 1.4. Let $X$ be an $H$ space. Then there are atomic spaces $X_{i}$ such that $X$ is homotopy equivalent to the weak direct product of the $X_{i}$ :

$$
X \simeq \prod X_{i} .
$$

Furthermore, the $X_{i}$ are unique up to order and do not depend on the $H$ space structure of $X$.

Corollary 1.5. Let $X$ be a simply-connected co-H space. Then there are atomic spaces $X_{i}$ such that $X$ is homotopy equivalent to the co-product of the $X_{i}$ in $\mathcal{C}_{p}^{\wedge}$ :

$$
X \simeq\left(\bigvee X_{i}\right)_{p}^{\wedge}
$$

Furthermore the $X_{i}$ are unique up to order and do not depend on the co- $H$ structure on $X$.

Proof. We consider Corollary 1.4 first. If $X$ is not atomic, choose a nontrivial idempotent self map by Theorem 1.3 . Write Tel $(e)$ for the telescope:

$$
X \stackrel{e}{\longrightarrow} X \stackrel{e}{\longrightarrow} X \stackrel{e}{\longrightarrow} \cdots,
$$

and similarly for $\operatorname{Tel}(1-e)$, where the $H$ space structure and inverse map are used to construct $1-e: X \longrightarrow X$. Then the natural map

$$
X \longrightarrow \operatorname{Tel}(e) \times \operatorname{Tel}(1-e)
$$

induces isomorphisms in all homotopy groups. Thus both telescopes are retracts of $X$ and so are themselves complete. Thus every nonatomic $H$ space can be split as a product. Since $X$ is of finite type, iteration of this process converges, and the limit is complete. The uniqueness assertion follows from the following argument essentially due to Wilkerson [W, 1.6].

Lemma 1.6. Suppose $X$ is atomic and $X$ is a retract of $X_{1} \times X_{2}$ where $X_{1}$ and $X_{2}$ are $H$ spaces. Then either $X$ is a retract of $X_{1}$ or a retract of $X_{2}$.

Proof. Let $e_{i}: X_{1} \times X_{2} \longrightarrow X_{1} \times X_{2}$ be projection through $X_{i}, i=1,2$. Then $\left(e_{1}\right)_{*}+\left(e_{2}\right)_{*}=1$ in homotopy. Let $f_{i}=X \longrightarrow X$ be the composition

$$
X \longrightarrow X_{1} \times X_{2} \stackrel{e_{i}}{\longrightarrow} X_{1} \times X_{2} \longrightarrow X .
$$

So $\left(f_{1}\right)_{*}+\left(f_{2}\right)_{*}=1$. This implies that $\left(f_{1}\right)_{*}\left(f_{2}\right)_{*}=\left(f_{2}\right)_{*}\left(f_{1}\right)_{*}$. So $1=\left[\left(f_{1}\right)_{*}+\right.$ $\left.\left(f_{2}\right)_{*}\right]^{N}=\sum\left(\begin{array}{c}N \\ i\end{array}\right)\left(f_{1}\right)_{*}^{i}\left(f_{2}\right)_{*}^{N-i}$. If both $f_{1}$ and $f_{2}$ were topologically nilpotent, this sum would be trivial for large $N$. Thus one of them is not topologically nilpotent and hence is an equivalence.

The argument for Corollary 1.5 is similar. We replace homotopy by homology, products by coproducts and use the co- $H$ structure to add and subtract. 
2.

We will often require that the space $T$ admits an $H$ space structure and the space $G$ admits a co- $H$ space structure, but will have no need to specify any particular structure. This is equivalent to assuming that the inclusion $\iota: T \longrightarrow \Omega S T$ has a left homotopy inverse and that the evaluation map $\epsilon: S \Omega G \longrightarrow G$ has a right homotopy inverse. For the later result we use the fact that $G$ is simply connected to conclude that the completion of the pullback square $\mathrm{Ga}$

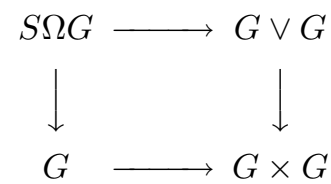

is again a pullback square. More generally we have (see [KSW]

Definition 2.1. A space $T$ is an $\mathcal{H}^{n}$ space if the inclusion $\iota: T \longrightarrow \Omega^{n} S^{n} T$ has a left homotopy inverse and a space $G$ is a co- $\mathcal{H}^{n}$ space if the evaluation $\epsilon: S^{n} \Omega^{n} G \longrightarrow G$ has a right homotopy inverse.

Theorem 2.2. There is a 1-1 correspondence between homotopy types of connected atomic $\mathcal{H}^{n}$ spaces and homotopy types of $n$-connected atomic co- $\mathcal{H}^{n}$ spaces for $n \geq$ 1.

Proof. Any $n$-fold suspension is a co- $\mathcal{H}^{n}$ space and retract of a co- $\mathcal{H}^{n}$ space is a co- $\mathcal{H}^{n}$ space. Thus, for any space $X$ we can apply Corollary 1.5 to $S^{n} X$ :

$$
S^{n} X \simeq \bigvee G_{i}
$$

and each $G_{i}$ is a co- $\mathcal{H}^{n}$ space. Choose one of these $G_{i}$ with the least connectivity. We shall see that in the case $X$ is a connected atomic $\mathcal{H}^{n}$ space, there is only one such choice. Similarly, if $X$ is $n$-connected choose an atomic factor $T$ of $\Omega^{n} X$ of least connectivity. We shall see that in the case that $X$ is an atomic co- $\mathcal{H}^{n}$ space, again, there is only one choice.

Now let us begin with an $n$-connected atomic co- $\mathcal{H}^{n}$ space $G$. Choose $T$ atomic of least connectivity as above. We have

$$
T \stackrel{g^{\prime}}{\longrightarrow} \Omega^{n} G \stackrel{h^{\prime}}{\longrightarrow} T
$$

where $h^{\prime} g^{\prime} \simeq 1$. Next choose $G^{\prime}$ atomic and of least connectivity:

$$
G^{\prime} \stackrel{f^{\prime \prime}}{\longrightarrow} S^{n} T \stackrel{g^{\prime \prime}}{\longrightarrow} G^{\prime}
$$

with $g^{\prime \prime} f^{\prime \prime} \simeq 1$. We now construct a homotopy equivalence between $G$ and $G^{\prime}$. This will complete the proof. The maps are:

$$
\begin{array}{cccccc}
\alpha: & G \stackrel{\nu}{\longrightarrow} & S^{n} \Omega^{n} G & \stackrel{S^{n} h^{\prime}}{\longrightarrow} & S^{n} T \stackrel{g^{\prime \prime}}{\longrightarrow} & G^{\prime}, \\
\beta: & G^{\prime} \stackrel{f^{\prime \prime}}{\longrightarrow} & S^{n} T & \stackrel{S^{n} g^{\prime}}{\longrightarrow} & S^{n} \Omega^{n} G \stackrel{\epsilon}{\longrightarrow} & G,
\end{array}
$$

where $\epsilon$ is the evaluation map and $\nu$ is any right inverse to $\epsilon$. We will show that both $\alpha \beta$ and $\beta \alpha$ are equivalences.

Suppose that $G$ is $(k+n-1)$ connected $(k \geq 1)$ and $\pi_{k+n}(G) \neq 0$. Then $\nu_{*}$ and $\epsilon_{*}$ are inverse isomorphisms between $\pi_{k+n}(G)$ and $\pi_{k+n}\left(S^{n} \Omega^{n} G\right)$. Since $\left(S^{n} h^{\prime}\right)\left(S^{n} g^{\prime}\right) \simeq 1$ and $g^{\prime \prime} f^{\prime \prime} \simeq 1$, it follows that $(\alpha \beta)_{*}: \pi_{k+n}\left(G^{\prime}\right) \longrightarrow \pi_{k+n}\left(G^{\prime}\right)$ is an isomorphism. $\Omega^{n} G$ is $k-1$ connected and $\pi_{k}\left(\Omega^{n} G\right) \neq 0$. By the choice of 
$T$ having minimal connectivity, $T$ is $k-1$ connected and $\pi_{k}(T) \neq 0$. Hence $S^{n} T$ is $k+n-1$ connected and $\pi_{k+n}\left(S^{n} T\right) \neq 0$. By the choice of $G^{\prime}$ having minimal connectivity, $\pi_{k+n}\left(G^{\prime}\right) \neq 0$. Therefore $\alpha \beta$ is not topologically nilpotent. Since $G^{\prime}$ is atomic, $\alpha \beta$ is an equivalence. Let $e=\beta \circ(\alpha \beta)^{-1} \circ \alpha: G \longrightarrow G$. Then $e$ is an idempotent. Since $G$ is atomic either $e$ is an equivalence or $e \sim *$. But $e$ induces an isomorphism in $\pi_{k+n}$, so $e$ is an equivalence and thus both $\alpha$ and $\beta$ are equivalences.

Two choices were made in this proof: first we chose $T$ from the factors of $\Omega^{n} G$ which had minimal connectivity. Then we chose $G^{\prime}$ from the factors of $S^{n} T$ which had minimal connectivity. However by choice, $\pi_{k+n}\left(G^{\prime}\right) \subseteq \pi_{k}(T) \subseteq \pi_{k+n}(G)$. Since $\alpha$ and $\beta$ are equivalences, these inclusions are equalities, and $G^{\prime}$ is the only factor of $S^{n} T$ which is not $k+n$ connected and $T$ is the only factor of $\Omega^{n} G$ which is not $k$ connected. In fact, we have

Corollary 2.3. In the correspondence $G \longleftrightarrow T$ between atomic $k+n-1$-connected co-H $\mathcal{H}^{n}$ spaces $G$ and atomic $k-1$-connected $\mathcal{H}^{n}$ spaces $T$, each of the maps in the commutative diagram

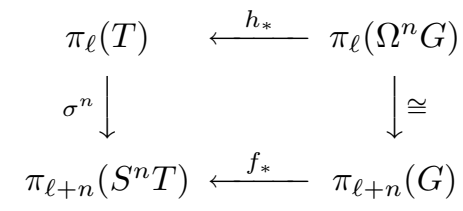

is an isomorphism for $\ell \leq 2 k-1$.

Definition 2.4. We call a pair of connected atomic spaces $(G, T)$ a corresponding pair if there are structure maps $f, g, g^{\prime}, h$ such that the composites

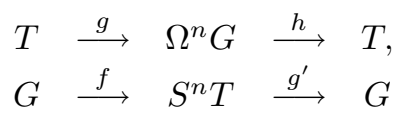

are the identity.

Proposition 2.5. Given a corresponding pair $(G, T)$ we may choose maps $f, g, g^{\prime}, h$ such that $g$ and $g^{\prime}$ are adjoint.

Proof. Given $f, g, g^{\prime}, h$, we will keep $g, h$ and replace $f$ with a map $\tilde{f}$ so that $g^{*} \cdot \tilde{f}=$ 1 , where $g^{*}$ is the adjoint of $g$. Since the composite

$$
G \stackrel{f}{\longrightarrow} S^{n} T \stackrel{g^{*}}{\longrightarrow} G
$$

induces an isomorphism in $\pi_{k+n}$ and $G$ is atomic, this composite is also a homotopy equivalence. Now define $\widetilde{f}=f \circ\left(g^{*} f\right)^{-1}$. Clearly we could also prove this result retaining $g^{\prime}$ and $f$ and replacing $h$ by a map $\widetilde{h}$ with the same effect.

Athough our main focus will be on the case $n=1$, at this point we will discuss some examples in the case $n=2$.

Example 2.6. $\Omega^{2} S^{3}\langle 3\rangle$ is an atomic $\mathcal{H}^{3}$ space, and the corresponding co- $\mathcal{H}^{3}$ space is $P^{2 p+2}=S^{2 p+1} \cup_{p \iota} e^{2 p+2}$; i.e., there are retractions:

$$
\begin{array}{cccc}
\Omega^{2} S^{3}\langle 3\rangle \stackrel{g}{\longrightarrow} \Omega^{3} P^{2 p+2} \stackrel{h}{\longrightarrow} \Omega^{2} S^{3}\langle 3\rangle, \\
P^{2 p+2} \stackrel{f}{\longrightarrow} S^{3} \Omega^{3} S^{3}\langle 3\rangle \stackrel{g^{\prime}}{\longrightarrow} P^{2 p+2} .
\end{array}
$$


In particular, $h=\Omega^{2} h^{\prime}$, where $h^{\prime}: \Omega P^{2 p+2} \longrightarrow S^{3}\langle 3\rangle$ is onto in homotopy. In fact, if $p>3$, it can be seen that $h^{\prime}$ factors through $S^{2 p+1}\{p\}$.

Proof. We first observe that $S^{3}\langle 3\rangle$ is $2 p-1$ connected and $\pi_{2 p}\left(S^{3}\langle 3\rangle\right)=\mathbb{Z} / p$. Thus we may construct a map $P^{2 p+1} \longrightarrow S^{3}\langle 3\rangle$ inducing an isomorphism in $\pi_{2 p}$. We use the $H$ space structure 2 on $S^{3}\langle 3\rangle$ to extend this map to a map $h^{\prime}: \Omega P^{2 p+2} \longrightarrow S^{3}\langle 3\rangle$, and define $h=\Omega^{2} h^{\prime}$. We construct $g$ using a lifting $H^{\prime}$ of the loops on the Hopf invariant map $\Omega^{2} S^{3} \stackrel{\Omega H}{\longrightarrow} \Omega^{2} S^{2 p+1}$ :

$$
\Omega^{2} S^{3} \stackrel{H^{\prime}}{\longrightarrow} \Omega^{2} S^{2 p+1}\{p\},
$$

where $S^{2 p+1}\{p\}$ is the fiber of the degree $p$ map on $S^{2 p+1}$ (see, for example, G4, $\S 4])$. There is a natural map $S^{2 p+1}\{p\} \stackrel{L}{\longrightarrow} \Omega P^{2 p+2}$ obtained from the obvious fibrations, and these maps combine to define $g$ :

$$
\Omega^{2} S^{3}\langle 3\rangle \longrightarrow \Omega^{2} S^{3} \stackrel{H^{\prime}}{\longrightarrow} \Omega^{2} S^{2 p+1}\{p\} \stackrel{\Omega^{2} L}{\longrightarrow} \Omega^{3} P^{2 p+2} .
$$

All of these maps induce isomorphism in $\pi_{2 p-2}$, and $\Omega^{2} S^{3}\langle 3\rangle=B W(1)$ is atomic. Since $\Omega^{2} S^{3}\langle 3\rangle$ is an $\mathcal{H}^{3}$ space and $P^{2 p+2}$ is a co- $\mathcal{H}^{3}$ space, we have proven the correspondence. Note that the corresponding map $f$ can be defined as the composition

$$
P^{2 p+2} \stackrel{\nu}{\longrightarrow} S^{3} \Omega^{3} P^{2 p+2} \stackrel{S^{3} h}{\longrightarrow} S^{3} \Omega^{2} S^{3}\langle 3\rangle
$$

so $f$ is a triple suspension and $h$ is a triple loop map.

One is tempted to generalize this. $\Omega^{2 n} S^{2 n-1}\langle 2 n+1\rangle$ is an $\mathcal{H}^{2 n+1}$ space, and one seeks to understand the corresponding co- $H^{2 n+1}$ space $G$. Note that the transfer defines a map

$$
S^{2 n+1} B^{n q} \stackrel{\lambda}{\longrightarrow} S^{2 n+1}\langle 2 n+1\rangle
$$

where $B^{n q}$ is the $n q$ skeleton of the $p$ localization of $B \Sigma_{p}$. We can extend this to a map

$$
h^{\prime}: \Omega S^{2 n+2} B^{n q} \longrightarrow S^{2 n+1}\langle 2 n+1\rangle .
$$

This gives a candidate for $h$ :

$$
h=\Omega^{2 n} h^{\prime}: \Omega^{2 n+1} S^{2 n+2} B^{n q} \longrightarrow \Omega^{2 n} S^{2 n+1}\langle 2 n+1\rangle
$$

and $f=S^{2 n+1} f^{\prime}$

$$
f^{\prime}: S B^{n q} \longrightarrow \Omega^{2 n} S^{2 n+1} B^{n q} \stackrel{\Omega^{2 n} \lambda}{\longrightarrow} \Omega^{2 n} S^{2 n+1}\langle 2 n+1\rangle .
$$

Constructing $G$ is not easy, and what we can see is that the composite

$$
S^{2 n+2} B^{n q} \stackrel{f}{\longrightarrow} S^{2 n+1} \Omega^{2 n} S^{2 n+1}\langle 2 n+1\rangle \longrightarrow G
$$

is a monomorphism in homology. $G$ may, however, be somewhat larger.

Another example is provided by Neisendorfer's result [N]: If $P^{2 n+1}\left(p^{r}\right)=S^{2 n} \cup_{p^{r}}$ $e^{2 n+1}$ and $r \geq 2$, then $\Omega^{2} P^{2 n+1}\left(p^{r}\right)$ contains, as an atomic factor, the fiber $D(n, p)$ of the map $\Omega^{2} S^{2 n+1} \stackrel{\pi}{\longrightarrow} S^{2 n-1}$ of degree $p^{r}$ constructed in [CMN]. Thus $D(n, r) \leftrightarrow$ $P^{2 n+1}\left(p^{r}\right)$ is a correspondence of an atomic $\mathcal{H}^{2}$ space $D(n, r)$ and an atomic co- $\mathcal{H}^{2}$ space $P^{2 n+1}\left(p^{r}\right)$ for $r \geq 2$.

\footnotetext{
${ }^{2} h^{\prime}$ is actually a loop map since $S^{3}\langle 3\rangle$ is a loop space.
} 
3.

Let us return to the case $n=1$. Let $(G, T)$ be a corresponding pair, and choose structure maps $(f, g, h)$ as in section 2 with $g^{\prime}$ adjoint to $g$. These maps determine an $H$ space structure on $T$ and a co- $H$ space structure on $G$ as follows:

$$
\begin{array}{cccccccc}
m: & T \times T & \stackrel{g \times g}{\longrightarrow} & \Omega G \times \Omega G & \longrightarrow & \Omega G & \stackrel{h}{\longrightarrow} & T, \\
n: & G & \stackrel{f}{\longrightarrow} & S T & \longrightarrow & S T \vee S T & \stackrel{g^{\prime} \vee g^{\prime}}{\longrightarrow} & G \vee G .
\end{array}
$$

In the pull back diagram

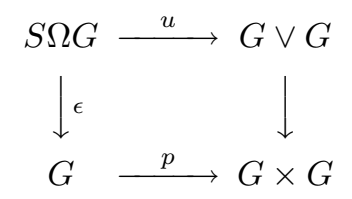

$u$ is the composition $S \Omega G \longrightarrow S \Omega G \vee S \Omega G \stackrel{\epsilon \vee \epsilon}{\longrightarrow} G \vee G$. Consequently the right inverse corresponding to $n$ is the composition

$$
\nu: G \stackrel{f}{\longrightarrow} S T \stackrel{s g}{\longrightarrow} S \Omega G .
$$

The case of the $H$ space structure maps is more complicated. In the absence of homotopy associativity, two nonhomotopic maps $\mu_{1}, \mu_{2}: \Omega S T \longrightarrow T$ can yield the same $H$ space structure map $m$ when composed with

$$
T \times T \longrightarrow \Omega S T \times \Omega S T \longrightarrow \Omega S T .
$$

However, one such choice is the composition

$$
\Omega S T \stackrel{\Omega g^{\prime}}{\longrightarrow} \Omega G \stackrel{h}{\longrightarrow} T .
$$

At this point we introduce a "Hopf fibration" sequence for $T$. This can be done for any connected $H$ space either with the classical Hopf construction

$$
H(m): T * T \longrightarrow S T
$$

(Sugawara [S]) or via a construction of Dold-Lashof construction ([DL], G2]):

$$
H_{m}: T * T \simeq E_{m} \longrightarrow S T .
$$

These constructions are different 3 , and we will find the Dold-Lashof construction advantageous. Specifically we will use the following corollary of Propositions A1 and A2 of the Appendix.

Corollary 3.1. Suppose $T$ is atomic. Then there is a 1-1 correspondence between fiber homotopy classes of fibrations

$$
T \stackrel{i}{\longrightarrow} E \stackrel{H}{\longrightarrow} S T
$$

with $i$ null homotopic and homotopy classes of maps

$$
m: T \times T \longrightarrow T
$$

such that:

a) $m(*, t)=t$,

b) the map $f: T \longrightarrow T$ given by $f(t)=m(t, *)$ is a homotopy equivalence. This correspondence is given by $H=H_{m}$.

\footnotetext{
${ }^{3}$ We thank Yukata Hemmi and Norio Iwase for helpful e-mail notes at this point.
} 
Proof. The only part that does not follow immediately from A1 and A2 of the Appendix is that if $i$ is null homotopic, $f$ is a homotopy equivalence. $f$ is the composition

$$
T \longrightarrow \Omega S T \stackrel{\partial}{\longrightarrow} T
$$

where $\partial: \Omega S T \longrightarrow T$ is from the fiber sequence. If $i$ is null homotopic, $\partial$ has a right homotopy inverse. Hence $f_{*}: \pi_{k}(T) \longrightarrow \pi_{k}(T)$ is onto. Since $\pi_{k}$ is complete and finitely generated, $f_{*}: \pi_{k}(T) \otimes \mathbb{Z} / p^{r} \rightarrow \pi_{k}(T) \otimes \mathbb{Z} / p^{r}$ is an isomorphism for each $r$. Hence $f_{*}$ is an isomorphism and $f$ is a homotopy equivalence.

We now choose an arbitrary $H$ space structure $m: T \times T \longrightarrow T$ and a corresponding fibration using (3.1). We will later see that some improvements can be made if we can choose a homotopy associative $H$ space structure. Choose a map $f: G \longrightarrow S T$ which induces an isomorphism in $\pi_{k}$ and choose $h$ as the composite:

$$
\Omega G \stackrel{\Omega f}{\longrightarrow} \Omega S T \stackrel{\partial}{\longrightarrow} T,
$$

where $\partial$ comes from the fibration sequence. It may not be possible to choose $g^{\prime}$ adjoint to $g$ so that $h g=1$ and $g^{\prime} f=1$; however any choice of $g$ which induces an isomorphism in $\pi_{k-1}$ will yield that $h g$ and $g^{\prime} f$ are homotopy equivalences. We now construct a commutative diagram obtained by pulling back the fibration from (3.1) along the base:

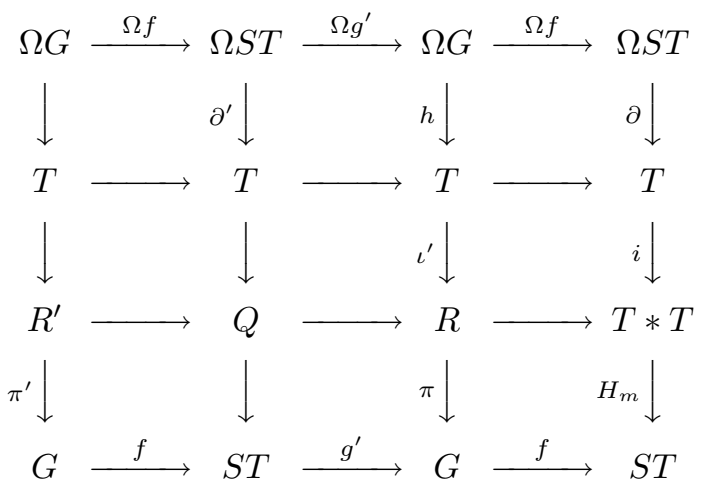

By Proposition A1 in the Appendix, $Q$ is determined by the restriction of the action map

$$
T \times T \longrightarrow \Omega S T \times T \stackrel{a}{\longrightarrow} T .
$$

Since $\partial^{\prime}=h \circ \Omega g^{\prime}$, the composition $T \longrightarrow \Omega S T \stackrel{\partial^{\prime}}{\longrightarrow} T$ is a homotopy equivalence, so $Q \simeq T * T$ By Corollary 3.1. Since $g^{\prime} f$ is a homotopy equivalence, $R^{\prime} \simeq R$ and $R$ is a retract of $T * T$. Since $h$ has a right homotopy inverse, $i^{\prime}$ is null homotopic and we have

Theorem 3.2. There is a homotopy equivalence

$$
\Omega G \simeq T \times \Omega R,
$$

where $R$ is a retract of $T * T$ and hence a co-H space. Write $R=\bigvee R_{\alpha}$ with $R_{\alpha}$ atomic. Then:

a) if $G=S X, R_{\alpha}$ is a retract of $S X^{(i)}$ for some $i \geq 2$,

b) if $G$ is homotopy is associative, $S^{j-1} R_{\alpha}$ is a retract of $G^{(j)}$ for some $j \geq 2$. 
We think of the exact sequence of spaces

$$
* \longrightarrow \Omega R \longrightarrow \Omega G \stackrel{\partial}{\longrightarrow} T \longrightarrow *
$$

as a minimal free presentation of $T$. Minimal since $G$ is atomic and free since the homology of both $\Omega R$ and $\Omega G$ are tensor algebras. However $\partial$ is not an $H$ map in general.

Proof. The only parts needing attention are the last two assertions. If $G=S T, R$ is a retract of $T * T$ which in turn is a retract of $\Omega S X * \Omega S X \simeq \bigvee S X^{(i)} \wedge X^{(j)}$. Part b) follows from the following theorem of Theriault.

Theorem 3.3 (Theriault [T]). Let $G$ be a simply-connected homotopy co-associative co-H space. Then

$$
S \Omega G \simeq \bigvee_{k=1}^{\infty} G(k)
$$

where $G(k)$ is a homotopy co-associative co-H space and $S^{k-1} G(k) \simeq G^{(k)}$.

Proof of Theorem 3.2b). We write $R$ as a retract of $\Omega G * \Omega G \simeq \Omega G \wedge(S \Omega G)$. It follows that $R$ is a retract of $\bigvee \Omega G \wedge G(k)$. Thus each $R_{\alpha}$ is a retract of $\Omega G \wedge G(k)$ for some $k$. But $\Omega G \wedge G(k)$ is a retract of $\Omega G \wedge S \Omega G(k) \simeq \bigvee_{\ell \geq 1} G(\ell) \wedge \Omega G(k)$; now

$$
S \Omega G(k) \simeq \bigvee_{j \geq 1} G(k, j)
$$

with $S^{j-1} G(k, j) \simeq G(k)^{(j)}$. Consequently $S^{j} R_{\alpha}$ is a retract of $G(\ell) \wedge G(k)^{(j)}$ and $S^{\ell+k j-1} R_{\alpha}$ is a retract of $S^{(k-1) j} G^{(\ell)} \wedge G(k)^{(j)} \simeq G^{(\ell+k j)}$.

There is another sense in which the $H$-space $T$ is generated by $G$, namely:

Proposition 3.4. The image of the homomorphism

$$
f_{*}: \widetilde{H}_{i+1}(G) \longrightarrow \widetilde{H}_{i+1}(S T) \simeq \widetilde{H}_{i}(T)
$$

generates the possibly nonassociative ring $\widetilde{H}_{*}(T)$.

As an example, consider $T_{0}^{2 n+1} \subset \Omega P^{2 n+1}$, the atomic factor of [CMN]. Then $H_{*}\left(T_{0}^{2 n+1}\right)$ is generated by $u \in H_{2 n-1}\left(T_{0}^{2 n+1}\right)$ and $r \in H_{2 n}\left(T_{0}^{2 n+1}\right)$ as a nonassociative ring.

Proof. Let $R \subset \widetilde{H}_{*}(T)$ be the subing generated by the image of $f_{*}$. We first observe that the composition

$$
a^{\prime}: \Omega G \times T \stackrel{\Omega f \times 1}{\longrightarrow} \Omega S T \times T \stackrel{a}{\longrightarrow} T
$$

defines a $H_{*}(\Omega G)$ module structure on $\widetilde{H}_{*}(T)$.

Lemma 3.5. $R$ is a $H_{*}(\Omega G)$ submodule.

Proof. The map $a^{\prime}$ fits into a commutative diagram

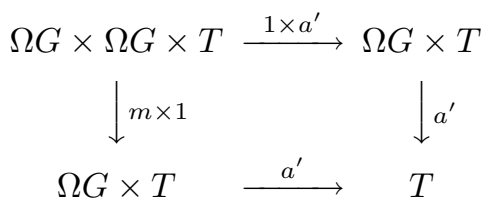


which can be obtained from Proposition A3 of the Appendix and the definition of $a^{\prime}$. Thus

$$
\left(a^{\prime}\right)_{*}\left(\alpha_{1} \alpha_{2} \otimes t\right)=\left(a^{\prime}\right)_{*}\left(\alpha_{1} \otimes\left(a^{\prime}\right)_{*}\left(\alpha_{2} \otimes t\right)\right)
$$

for $\alpha_{1}, \alpha_{2} \in H_{*}(\Omega G)$ and $t \in H_{*}(T)$. Iterating we get

$$
\left(a^{\prime}\right)_{*}\left(\alpha_{1} \ldots \alpha_{k} \otimes t\right)=\left(a^{\prime}\right)_{*}\left(\alpha_{1} \otimes\left(a^{\prime}\right)_{*}\left(a_{2}, \otimes \ldots,\left(a^{\prime}\right)_{*}\left(\alpha_{k} \otimes t\right) \ldots\right)\right) .
$$

It follows that it is sufficient to show that $\left(a^{\prime}\right)_{*}(\alpha \otimes t) \in R$ whenever $t \in R$ and $\alpha$ is indecomposable. Since indecomposable elements of $H_{*}(\Omega G)$ are in the image of the homomorphism

$$
\widetilde{H}_{i}(G) \stackrel{\nu_{*}}{\longrightarrow} \widetilde{H}_{i}(S \Omega G) \simeq \widetilde{H}_{i-1}(\Omega G)
$$

and $\nu \sim S g \circ f$, any indecomposable element $\alpha$ can be written as $g_{*}(r)$ for some $r \in R$. Now we have a commutative diagram

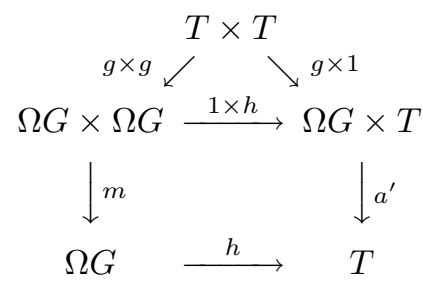

where the commutative square is a restriction of the above square to $\Omega G \times \Omega G \times * \subset$ $\Omega G \times \Omega G \times T$. Since the composition along the left and bottom is $m$, we have $a_{*}\left(g_{*}(r) \otimes t\right)=m_{*}(r \otimes t)=r t \in R$. This proves the lemma.

We now use this lemma to prove the proposition. Let $\xi \in H_{*}(T)$ be an element of least degree that is not in $R$. $\xi=h_{*}(\alpha)$ for some $\alpha \in H_{*}(\Omega G)$. $\alpha$ cannot be indecomposable since then it would be in the image of $\nu_{*}$ and hence $\xi$ would be in the image of $f_{*}$. Thus $\alpha=\Sigma \alpha_{i}^{\prime} \alpha_{i}^{\prime \prime}$ with $\alpha_{i}^{\prime}$ and $\alpha_{i}^{\prime \prime}$ of positive degree. In particular $\xi=h_{*}\left(\Sigma \alpha_{i}^{\prime} \alpha_{i}^{\prime \prime}\right)=\Sigma\left(a^{\prime}\right)_{*}\left(\alpha_{i}^{\prime} \otimes h_{*}\left(\alpha_{i}^{\prime \prime}\right)\right)$. Since $\operatorname{deg} h_{*}\left(\alpha_{i}^{\prime \prime}\right)<\operatorname{deg} \xi, h_{*}\left(\alpha_{i}^{\prime \prime}\right) \in R$ and hence, by the lemma $\xi \in R$.

Proposition 3.6. If $G$ is homotopy co-commutative, and co-associative each element in imf $f_{*}$ is primitive (and hence $H_{*}(T)$ is primitively generated). Conversely, if, in addition $T$ is homotopy associative and homotopy commutative, each primitive element in $H_{*}(T)$ is a sum of $p^{r}$ th the powers of elements in imf $f_{*}$.

This depends on the following lemma which has been noted by $[\mathrm{T}$ as a corollary of the results of Berstein $[\mathrm{B}]$. The proof in $[\mathrm{B}]$ is not direct, and we offer here a direct proof.

Lemma 3.7. If $G$ is co-commutative co-associative co- $H$ space, the image of

$$
\nu_{*}: \widetilde{H}_{i}(G) \longrightarrow \widetilde{H}_{i-1}(\Omega G)
$$

consists of primitive elements.

Proof. We first examine the case that $G=S X$ for some $X$. Consider the composite

$$
\Gamma_{X}: S X \stackrel{S \Delta}{\longrightarrow} S X \wedge X \stackrel{W}{\longrightarrow} S X \vee S X
$$

where $\Delta$ is the diagonal and $W$ is the Whitehead product. By definition, this is $\iota_{1}+\iota_{2}-\iota_{1}-\iota_{2}$, where $\iota_{1}, \iota_{2}: S X \longrightarrow S X \vee S X$ are the inclusions. This can be written as $\left(\iota_{1}+\iota_{2}\right)-\left(\iota_{2}+\iota_{1}\right)=\phi-\tau \phi$, where $\phi: S X \longrightarrow S X \vee S X$ is the usual 
co- $H$ structure map. In particular, the composite $W \circ S \Delta \sim *$ iff the usual co- $H$ structure is co-commutative. Now consider the composite

$$
G \stackrel{\nu}{\longrightarrow} S \Omega G \stackrel{\Gamma_{\Omega G}}{\longrightarrow} S \Omega G \vee S \Omega G \stackrel{\epsilon \vee \epsilon}{\longrightarrow} G \vee G
$$

Since $G$ is co-associative, $\nu$ is a co- $H$ map and hence this composition is trivial in the case that $G$ is co-commutative. However we further factor this as

$$
G \stackrel{\nu}{\longrightarrow} S \Omega G \stackrel{S \Delta}{\longrightarrow} S(\Omega G \wedge \Omega G) \stackrel{W}{\longrightarrow} S \Omega G \vee S \Omega G \stackrel{\epsilon \vee \epsilon}{\longrightarrow} G \vee G .
$$

The composite $(\epsilon \vee \epsilon) W$ is the inclusion of the fiber in the fibering

$$
S(\Omega G \wedge \Omega G) \longrightarrow G \vee G \longrightarrow G \times G
$$

since this inclusion has a null homotopic fiber, we conclude that the composite

$$
G \stackrel{\nu}{\longrightarrow} S \Omega G \stackrel{S \Delta}{\longrightarrow} S(\Omega G \wedge \Omega G)
$$

is null homotopic from which the result follows.

Proof of Proposition 3.6. Since $f: G \rightarrow S T$ is the composition $G \stackrel{\nu}{\longrightarrow} S \Omega G \stackrel{S h}{\longrightarrow}$ $S T$, the first part is immediate. However, if $\xi \in H_{*}(T)$ is primitive, so is $g_{*}(\xi) \in$ $H_{*}(\Omega G)$. Since $H_{*}(\Omega G)$ is a primitively generated tensor algebra, $g_{*}(\xi)$ is a sum of $p^{r}$ th powers of commutators $(r \geq 0)$ and $p^{r}$ th powers of elements in the image of $\nu_{*}$. But if $T$ is homotopy associative and homotopy commutative, $\xi=h_{*} g_{*}(\xi)$ is a sum of $p^{r}$ th powers of elements in $i m f_{*}$.

We now derive a result from the following theorem of Theriault $\mathrm{T}$, Theorem 8.4].

Theorem 3.8 (Theriault). If $G$ is a homotopy co-commutative and co-associative co-H space and $(l, p)=1$, there is a decomposition

$$
\Omega G=\Omega U_{l} \times F_{l}
$$

induced by a map $\phi_{l}: U_{l} \longrightarrow G$, where the homology of $\Omega U_{l}$ is the subalgebra of the homology of $\Omega G$ generated by the commutators of length $l$.

Corollary 3.9. For some $H$ space structure on $T$, the image in homology of $\Omega \pi$ : $\Omega R \rightarrow \Omega G$ contains all commutators of length $l$ where $(l, p)=1$.

Proof. $\Omega U_{l}$ is homotopy equivalent to a product of some of the factors of $\Omega G$. Since $\Omega U_{l}$ is $l k-1$ connected, $T$ is not among them. These factors lie in $\Omega R$, and we replace them by $\Omega U_{l}$, for each $l$ with $(l, p)=1$, and take $T$ to be the fiber of this new map. This new $T$ will still be the bottom atomic factor of $\Omega G$, but the map from $\Omega G$ to $T$ and hence the $H$ space structure may be different. See Example 3.13 below.

$G$ is a generator for $T$ in another sense as well:

Proposition 3.10. Let $X$ be any $H$ space and let $\psi_{i}: T \rightarrow X$ be $H$ maps for $i=1,2$. Then:

a) $\psi_{1} \sim \psi_{2} \Longleftrightarrow\left(S \psi_{1}\right) f \sim\left(S \psi_{2}\right) f$

$$
G \stackrel{f}{\longrightarrow} S T \stackrel{S \psi_{i}}{\longrightarrow} S X \text {. }
$$

b) If, in addition, $G=S K$ and $f=S \bar{f}$ for $\bar{f}: K \rightarrow T$, then

$$
\psi_{1} \sim \psi_{2} \text { iff } \psi_{1} \bar{f} \sim \psi_{2} \bar{f} .
$$


Proof. From the commutative diagram

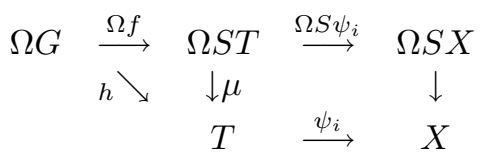

we see that if $\left(S \psi_{1}\right) f \sim\left(S \psi_{2}\right) f, \psi_{1} h \sim \psi_{2} h$ so $\psi_{1} \sim \psi_{1} h g \sim \psi_{2} h g \sim \psi_{2}$. If $f=S \bar{f}$, $\left(S \psi_{1}\right) f \sim\left(S \psi_{2}\right) f$ iff $S\left(\psi_{1} \bar{f}\right) \sim S\left(\psi_{2} f\right)$. Since $X$ is an $H$ space, this is true iff $\psi_{1} f \sim \psi_{2} f$.

There are numerous examples in the literature of spaces $K$ with this property ([CMN], G1], $\mathrm{Se}]$ ).

Write $P_{X}(t)$ for the Poincaré series for $X$.

Proposition 3.11. $P_{R}=P_{T} P_{G}-(t+1)\left(P_{T}-1\right)$.

Proof. Since $\Omega G=\Omega R \times T$, we have

$$
\frac{P_{T}}{1-t^{-1}\left(P_{R}-1\right)}=\frac{1}{1-t^{-1}\left(P_{G}-1\right)}
$$

which implies the result.

Corollary 3.12. If $G=S X$ and $G \wedge G \cong \bigvee_{\alpha \in A} S^{n_{\alpha}} G$, then $R \simeq G \wedge W$, where $W$ is a wedge of spheres and

$$
P_{W}=P_{T}-\frac{t\left(P_{T}-1\right)}{P_{G}-1}+1 .
$$

Furthermore if $T_{n}$ corresponds to $S^{n} X$ for each $n \geq 1, \Omega S X \simeq \prod T_{n_{i}}$ for some sequence $n_{i}$.

Proof. The hypothesis implies that $R=\bigvee R_{\alpha}$, where each $R_{\alpha}$ is a suspension of $G$ by Theorem 3.2. Thus $R \simeq G \wedge W$, where $W$ is a wedge of spheres. The formula for $P_{W}$ follows from Proposition 3.11. Applying the Hilton-Milnor theorem and induction decomposes the loop space of the wedge into a product.

In the next example we see that the map $\pi$ does depend on the $H$ space structure.

Example 3.13. Let $T=S^{2 p-3}\{p\}$, the fiber of the degree $p$ map on $S^{2 p-3}$. We will write $P^{n}=S^{n-1} \cup_{p \iota} e^{n}$. Then $G=P^{2 p-2}$, and $R=\bigvee R_{\alpha}$, where each $R_{\alpha}=P^{n_{\alpha}}$ by Corollary 3.12. In fact, $R=\bigvee_{k \geq 1} P^{2 p-1+k(2 p-4)}$.

One way of obtaining this decomposition is given in [CMN], where the maps $P^{2 p-1+k(2 p-4)} \longrightarrow P^{2 p-2}$ are given by iterated Whitehead products. In particular $\pi$ is stably inessential. On the other hand, let $B=\left(B \Sigma_{p}\right)_{(p)}$. There is a fibration sequence

$$
\Omega B \longrightarrow S^{2 p-3} \stackrel{p}{\longrightarrow} S^{2 p-3} \longrightarrow B .
$$

Since $\Omega B \simeq S^{2 p-3}\{p\}$, this determines another $H$ space structure on $S^{2 p-3}\{p\}$. There is a pull-back diagram

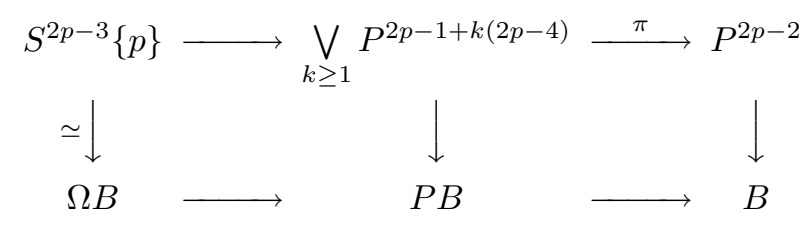


from which we can see that the composition

$$
\bigvee_{k \geq 1} P^{2 p-1+k(2 p-4)} \longrightarrow P^{2 p-2} \longrightarrow B
$$

is null homotopic. In particular, with $k=1$, the restriction of $\pi$ to $P^{4 p-5}$ is the attaching map for the next two cells of $B$. But the $4 p-4$ skeleton of $B$ is $P^{2 p-2} \cup C P^{4 p-5}$, and the Steenrod operation $\mathcal{P}^{1}$ is nonzero. Thus $P^{4 p-5} \longrightarrow P^{2 p-2}$ is stably essential. In particular, the Whitehead product map $P^{4 p-5} \longrightarrow P^{2 p-2}$ does not factor through $R$, and the commutator $P^{4 p-6} \longrightarrow \Omega P^{2 p-2} \longrightarrow S^{2 p-3}\{p\}$ is nontrivial.

We now consider the special case when there is a homotopy associative multiplication on $T$.

Proposition 3.14. Suppose there is a homotopy associative multiplication on $T$. Then in diagram (A), $\partial=\partial^{\prime}: \Omega S T \rightarrow T$ is an $H$ map, $h$ is an $H$ map, the fiberings $Q \longrightarrow S T$ and $R \longrightarrow S T$ are fiber homotopy equivalent, $g$ can be chosen so that $h g \sim 1$ and $g^{\prime} f \sim 1$, and $\pi$ is the composition

$$
R \longrightarrow T * T \stackrel{H_{m}}{\longrightarrow} S T \stackrel{g^{\prime}}{\longrightarrow} G .
$$

Proof. We show that there are structure maps $(f, g, h)$ so that the composition $\Omega S T \stackrel{S g^{\prime}}{\longrightarrow} \Omega G \stackrel{h}{\longrightarrow} T$ factors the given map $\mu: \Omega S T \longrightarrow T$ extending the multiplication. To do this, we choose any of the structure maps $\left(f_{0}, g_{0}, h_{0}\right)$. We begin with the composition

$$
e: T \stackrel{g_{0}}{\longrightarrow} \Omega G \stackrel{\Omega f_{0}}{\longrightarrow} \Omega S T \stackrel{\mu}{\longrightarrow} T,
$$

where $e$ induces an isomorphism in $\pi_{k}$, so it is a homotopy equivalence. Define $g=g_{0}\left(e^{-1}\right)$ and $h=\mu\left(\Omega f_{0}\right)$. Then $h g=1$. Now the composition

$$
e^{\prime}: G \stackrel{f_{0}}{\longrightarrow} S T \stackrel{g^{\prime}}{\longrightarrow} G
$$

is a homotopy equivalence where $g^{\prime}$ is the adjoint of $g$. Let $f=f_{0}\left(e^{\prime}\right)^{-1}$. Then $g^{\prime} f \sim 1$. To show that these structure maps determine $\mu$, define $\bar{\mu}$ as the indicated composite:

$$
\bar{\mu}: \Omega S T \stackrel{\Omega g^{\prime}}{\longrightarrow} \Omega G \stackrel{\Omega f_{0}}{\longrightarrow} \Omega S T \stackrel{\mu}{\longrightarrow} T .
$$

Since $\mu$ is an $H$ map, $\bar{\mu}$ is as well. To see that $\mu \sim \bar{\mu}$ we need only calculate $\left.\bar{\mu}\right|_{T}=\mu\left(\Omega f_{0}\right) g=h g \sim 1$. By Proposition A3 of the Appendix, $\partial \sim \mu$ and $\partial^{\prime}=h\left(\Omega g^{\prime}\right) \sim \mu$. The fibering $Q \longrightarrow S T$ is determined by the restriction of the action map

$$
T \times T \longrightarrow \Omega S T \times T \stackrel{a^{\prime}}{\longrightarrow} T,
$$

where $a^{\prime}$ is the composite

$$
\Omega S T \times T \stackrel{\Omega f \times 1}{\longrightarrow} \Omega G \times T \stackrel{\Omega g^{\prime} \times 1}{\longrightarrow} \Omega S T \times T \stackrel{a}{\longrightarrow} T .
$$

Since $T$ is homotopy associative, the formula in Proposition A3 implies that $a$ is the composite

$$
\Omega S T \times T \stackrel{\mu \times 1}{\longrightarrow} T \times T \longrightarrow T
$$

combining these we see that $a^{\prime}$ is the composite

$$
\Omega S T \times T \stackrel{\Omega f \times 1}{\longrightarrow} \Omega G \times T \stackrel{h \times 1}{\longrightarrow} T \times T \stackrel{m}{\longrightarrow} T,
$$

and restricting to $T \times T$ is thus $m$. 
4.

Given the strong duality involved here, it is natural to ask whether a dual discussion can be obtained. This would require a dual to the Hopf fibering. The following Conjecture is due to Ganea $\mathrm{Ga}$.

Conjecture 4.1. Given a co-H space $G$ there is a cofibration sequence

$$
\Omega G \stackrel{\sigma}{\longrightarrow} X \longrightarrow G \stackrel{\nu}{\longrightarrow} S \Omega G .
$$

This is certainly the case when $G$ is a suspension, for if $G=S A$ we can take $X=\Omega S A / A$. This is false in general.

Example 4.2. Let $\alpha_{1}: S^{2 p} \longrightarrow S^{3}$ be the first element of order $p$. Harper [H] has shown that $\alpha$ is a co- $H$ map and hence $G=S^{3} \cup_{\alpha} e^{2 p+1}$ is a co- $H$ space. Suppose such a space $X$ does exist. $H^{*}(\Omega G)$ through dimension $2 p$ has classes $u, u^{2}, \ldots, u^{p}$ and $v$ with $|u|=2$ and $|v|=2 p$. In $p$-local cohomology, the class $u^{p}-p v$ transgresses to the class in dimension $2 p+1$ in the base. Since $p>3$, the classes $u^{2}$ and $u^{p-2}$ are in the image of $\sigma^{*}$. Hence $u^{p}$ is in the image of $\sigma^{*}$. This is impossible since, $\bmod p, u^{p}$ transgresses.

This example is not a co-associative co- $H$ space (see $[\underline{\mathrm{H}}$ ), and it is conceivable that a co-Hopf construction is possible in the case $G$ is a co-associative. The requirement that $p>3$ is essential in this example.

Proposition 4.3. Suppose $S^{2} Y \stackrel{f}{\longrightarrow} S^{2 n+1}$ is a co-H map localized at 3 and $n \geq 1$, and set $G=S^{2 n+1} \cup_{f} C S^{2} Y$. Then there is a Hopf co-fibration

$$
\Omega G \stackrel{\sigma}{\longrightarrow} X \longrightarrow G \stackrel{\nu}{\longrightarrow} S \Omega G .
$$

The proof of Proposition 4.3 depends on the following

Lemma 4.4. Let $\widehat{S}^{2 n}=S^{2 n} \cup e^{4 n} \cup \cdots \cup e^{2 n(p-1)} \subset\left(S^{2 n}\right)_{\infty}$. Then the loops on the projection map

are null homotopic.

$$
\Omega \widehat{S}^{2 n} \longrightarrow \Omega S^{2 n(p-1)}
$$

Proof. Let $K=S^{2 n-1} \cup_{w_{n}} e^{2 n p-2}$, where $w_{n}$ is the first element in the kernel of the double suspension localized at $p$. Then there is a loop map $\Omega S K \longrightarrow \Omega \widehat{S}^{2 n}$ which has a right homotopy inverse [G1], so it suffices to show that the composite

$$
\Omega S K \longrightarrow \Omega \widehat{S}^{2 n} \longrightarrow \Omega S^{2 n(p-1)}
$$

is null homotopic. We will show that the composite $S K \longrightarrow \widehat{S}^{2 n} \longrightarrow S^{2 n(p-1)}$ is null homotopic. The degree $k$ map on $S^{2 n}$ induces a map $[k]: \widehat{S}^{2 n} \longrightarrow \widehat{S}^{2 n}$, and there are commutative diagrams

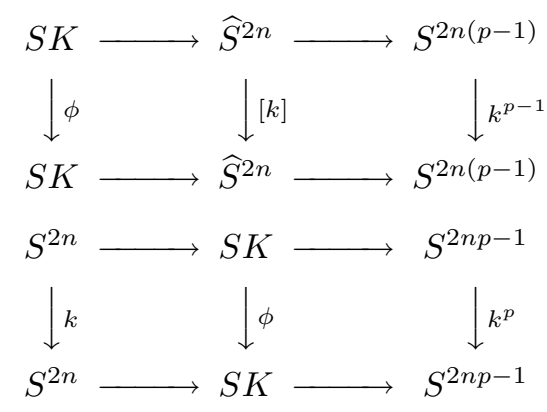


Since $S^{2} w_{n} \sim 0$, the composite $S K \longrightarrow \widehat{S}^{2 n} \longrightarrow S^{2 n(p-1)}$ factors uniquely over $S^{2 n p-1}$ :

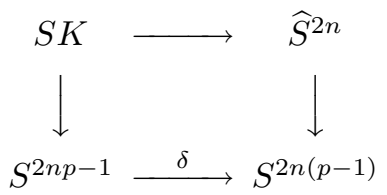

and these diagrams, together with the uniqueness, imply that $k^{p-1} \delta=\delta k^{p}$. Since $\delta$ must be a suspension, we conclude that $\left(k^{p}-k^{p-1}\right) \delta=0$. Let $k=-1$ to get $-2 \delta=0$ or $\delta=0$.

Proof of Proposition 4.3. According to Harper $[\underline{\mathrm{H}}]$, the Hopf invariant of $f$ is the co- $H$ deviation and hence is null homotopic. Therefore we can factor

$$
\begin{aligned}
S Y & \stackrel{f^{\prime}}{\longrightarrow} \Omega S^{2 n+1} \\
& \searrow{ }^{\uparrow} \\
& \bar{f} \widehat{S}^{2 n}=S^{2 n} \cup_{[\iota, \ell]} e^{4 n}
\end{aligned}
$$

Consequently $\Omega\left(S^{2 n+1} \cup_{f} C S^{2} Y\right)$ contains as a subcomplex $\widehat{S}^{2 n} \cup_{\bar{f}} C S Y$. Then

$$
\Omega\left(S^{2 n+1} \cup_{f} C S^{2} Y\right) / S^{2 n}
$$

contains $S^{4 n} \cup_{\tilde{f}} C S Y$, where $\tilde{f}$ is the projection of $\bar{f}$ onto $S^{4 n}$. However $\tilde{f}$ is null homotopic since $\Omega j: \Omega \widehat{S}^{2 n} \longrightarrow \Omega S^{4 n}$ is null homotopic. Thus $\Omega\left(S^{2 n+1} \cup_{f} C S^{2} Y\right) / S^{2 n}$ contains $\Omega\left(S^{4 n} \vee S^{2} Y\right)$. Let $X=\Omega\left(S^{2 n+1} \cup_{f} C S^{2} Y\right) / S^{2 n} / S^{2} Y$ and $\sigma$ be the projection $\Omega\left(S^{2 n+1} \cup_{f} C S^{2} Y\right) \longrightarrow X$. In homology $\sigma_{*}$ is onto and its kernel consists of all tensors of length 1 . Let $C$ be the cofiber of $\sigma$. Then $H_{*}(C) \longrightarrow H_{*}(S \Omega G)$ consists of the suspension of the tensors of length 1 and hence the composite

$$
C \longrightarrow S \Omega G \stackrel{\epsilon}{\longrightarrow} G
$$

is a homotopy equivalence, and we are done.

5.

We need to say something about naturality. Given corresponding pairs $\left(G_{1}, T_{1}\right)$, $\left(G_{2}, T_{2}\right)$ with structure maps $\left(f_{1}, g_{1}, h_{1}\right)$ and $\left(f_{2}, g_{2}, h_{2}\right)$, a map from $\left(G_{1}, T_{1}\right)$ to $\left(G_{2}, T_{2}\right)$ should be a pair of maps $(\phi, \psi)$ so that all diagrams commute. This would guarantee that $\phi$ is a co- $H$ map and $\psi$ is an $H$ map. In many situations we may begin with an $H$ map $\psi: T_{1} \longrightarrow T_{2}$, and it is not possible to find an appropriate co- $H$ map $\phi$. For example, $\psi=\Omega \gamma$, where $\gamma: S^{2 n+1} \longrightarrow S^{2 m+1}$ is not a co- $H$ map.

The following is the strongest result that seems reasonable.

Proposition 5.1. Suppose $\psi: T_{1} \rightarrow T_{2}$ is an $H$ map and $\phi: G_{1} \rightarrow G_{2}$ is a co- $H$ map. Then the following are equivalent:

a) $\phi$ is the composition $G_{1} \stackrel{f_{1}}{\longrightarrow} S T_{1} \stackrel{S \psi}{\longrightarrow} S T_{2} \stackrel{g_{2}^{\prime}}{\longrightarrow} G_{2}$.

b) The following square commutes:

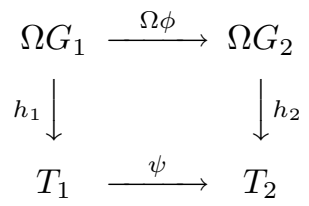


c) $\psi$ is the composition $T_{1} \stackrel{g_{1}}{\longrightarrow} \Omega G_{1} \stackrel{\Omega \phi}{\longrightarrow} \Omega G_{2} \stackrel{h_{2}}{\longrightarrow} T_{2}$.

d) The following square commutes:

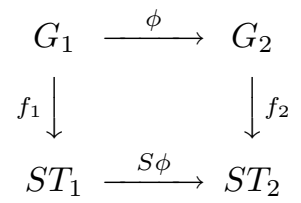

Proof. To see that a) implies b) note that $\mu_{i}=h_{i} \circ \Omega g_{i}^{\prime}$. So $h_{i} \sim \mu_{i} \circ \Omega f_{i}$; thus $\psi \circ h_{1} \sim \psi \circ \mu_{1} \circ \Omega f_{1} \sim \mu_{2} \circ \Omega S \psi \circ \Omega f_{1} \sim h_{2} \circ \Omega g_{2}^{\prime} \circ \Omega S \psi \circ \Omega f_{1}=h_{2} \circ \Omega \phi$. To see that b) implies c), compose the square with the map $T_{1} \stackrel{g_{1}}{\longrightarrow} \Omega G_{1}$. The last two parts are similar to the first two parts.

On the other hand if $T_{2}$ is homotopy associative b) implies that $\psi$ is an $H$ map, and if $G_{1}$ is homotopy co-associative d) implies that $\phi$ is a co- $H$ map.

6.

In this section we discuss a further refinement in the determination of $R$ in the case that $T$ has a homotopy associative $H$-space structure and $p>2$. The results are based on the following well-known result.

Lemma 6.1. Suppose $p>2$ and $\tau: X \rightarrow X$ is a map such that $\tau^{2} \sim 1$. Then $S X \simeq X_{+} \vee X_{-}$, where $\widetilde{H}_{*}\left(X_{ \pm}\right)=\left\{\xi \in \widehat{H}_{*}(S X) \mid \tau_{*}(\xi)= \pm \xi\right\}$.

Proof. Let $e_{ \pm}: S X \longrightarrow S X$ be given by $e_{ \pm}=\tau \pm 1$ and

$$
X_{ \pm}=\lim _{\longrightarrow}\left\{S X \stackrel{e_{ \pm}}{\longrightarrow} S X \stackrel{e_{ \pm}}{\longrightarrow} S X \longrightarrow \cdots\right\} \text {. }
$$

Then the composition $S X \longrightarrow S X \vee S X \longrightarrow X_{+} \vee X_{-}$induces homology isomorphisms between simply-connected spaces.

We will apply this lemma in two cases. We first consider the transposition map $\tau: T \wedge T \longrightarrow T \wedge T$, from which we write $T * T \simeq R_{+} \vee R_{-}$. The second application deals with the inverse map for a homotopy associative $H$-space. For any connected $H$-space, it is standard to construct left and right slicing maps

$$
\begin{aligned}
T \times T & \longrightarrow T, \\
(a, b) & \longrightarrow a / b, \\
(a, b) & \longrightarrow a \backslash b
\end{aligned}
$$

by choosing homotopy inverses for the maps

$$
\begin{gathered}
T \times T \longrightarrow T \times T, \\
(a, b) \longrightarrow(m(a, b), b), \\
(a, b) \longrightarrow(a, m(a, b)) .
\end{gathered}
$$

These maps define operations on the set of homotopy classes $\left[(X, *),\left(T_{1} e\right)\right]$ which satisfy the identities

$$
\begin{aligned}
(\alpha \cdot \beta) / \beta & =\alpha, \\
(\alpha / \beta) \cdot \beta & =\alpha, \\
\alpha \backslash(\alpha \beta) & =\beta, \\
\alpha(\alpha \backslash \beta) & =\beta .
\end{aligned}
$$


In the case $T$ is homotopy associative one can see that $e / \alpha=\alpha \backslash e$ and write $\alpha^{-1}$ for this homotopy class; then we have $\left(\alpha^{-1}\right)^{-1}=\alpha$ and $(\alpha \beta)^{-1}=\beta^{-1} \alpha^{-1}$. Write $\gamma: T \longrightarrow T$ for the inverse of the identify map. Then $\gamma^{2}=1$, so we have $S T \simeq T_{+} \vee T_{-}$.

If $\xi \in \widetilde{H}_{*}(T)$ is primitive, $\gamma_{*}(\xi)=-\xi$. Since $T$ is $k-1$ connected, all classes in $\widetilde{H}_{k}(T)$ are primitive. Consequently, the composition

$$
f_{0}: G \stackrel{f}{\longrightarrow} S T \longrightarrow T_{-} \longrightarrow S T
$$

is an isomorphism in dimension $k$. Now choose $g_{0}, h_{0}$ so that $g_{0}^{\prime} f_{0} \sim 1$ and $h_{0} g_{0} \sim 1$. We then apply the modification of Theorem 3.8 to obtain a triple $(f, g, h)$ with $\mu \sim h\left(\Omega g^{\prime}\right)$, and observe that $f: G \longrightarrow S T$ factors through $T_{-}$.

Proposition 6.2. The diagram

$$
\begin{gathered}
G \stackrel{f}{\longrightarrow} S T \\
\qquad \underset{\downarrow}{\downarrow} S T .
\end{gathered}
$$

commutes up to homotopy.

Proof. It suffices to show that $S \gamma\left(T_{ \pm}\right) \subset T_{ \pm}$and $\left.(-S \gamma)\right|_{T_{-}}=1$. This follows from the commutative ladders

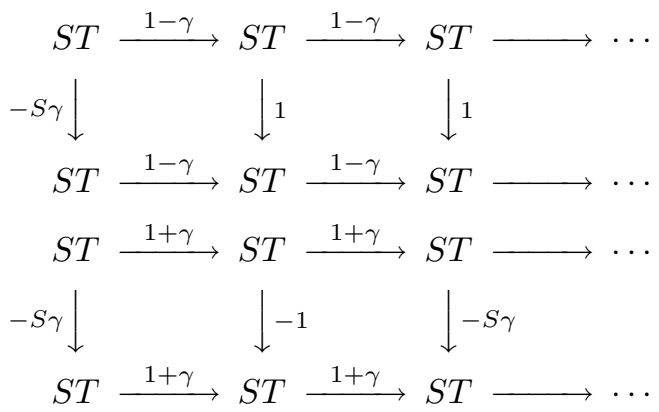

Theorem 6.3. The following diagram commutes up to homotopy:

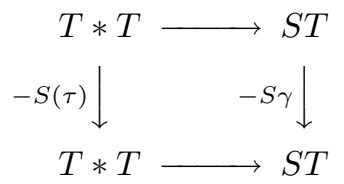

Corollary 6.4. $R$ is a retract of $R_{-} \subset T * T$.

Proof of Corollary 6.4. The diagram

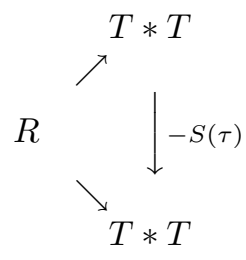

commutes after projection to $S T$ by Theorem 6.3. However the inclusion of the fiber $T \longrightarrow T * T$ is null homotopic, so this diagram actually commutes up to homotopy. As in the previous case, this implies that the projection onto $R_{+}$is null homotopic. 
Proof of Theorem 6.3. We begin by considering the equivalence $E_{m} \simeq T * T$ from Proposition A2 of the Appendix (where $A=F=T$ and $\theta=m$ ). From this we see that corresponding to $\pi_{1}$ in the push-out diagram for $E_{m}$ is the map $(a, b) \longrightarrow a / b$ in the push-out diagram for $T * T$. In other words, the map $E_{m} \longrightarrow S T$ corresponds to the map of push-out diagrams

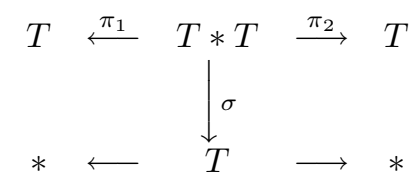

where $\sigma(a, b)=a / b$. This is, in fact, the classical Hopf construction on the map $\sigma$

$$
T * T \stackrel{H(\sigma)}{\longrightarrow} S T
$$

here we use the reduced join $X * Y$, which is the quotient of $X \times I \times Y$ under the identifications

$$
\begin{aligned}
(x, 0, y) & \sim\left(x, 0, y^{\prime}\right), \\
(x, 1, y) & \sim\left(x^{\prime}, 1, y\right), \\
(*, t, *) & \sim *
\end{aligned}
$$

and the reduced suspension. We introduce maps $\alpha: S(X \times Y) \longrightarrow X * Y$ and $\beta: X * Y \longrightarrow S(X \wedge Y)$ whose composite is homotopic to the quotient map $S(X \times Y) \longrightarrow S(X \wedge Y)$. We define $\alpha$ by

$$
\alpha(s, x, y)= \begin{cases}(x, 1-3 s, *), & 0 \leq s \leq 1 / 3 \\ (x, 3 s-1, y), & 1 / 3 \leq s \leq 2 / 3 \\ (*, 3-3 s, y), & 2 / 3 \leq s \leq 1\end{cases}
$$

and $\beta(x, t, y)=(t, x, y) . \quad \beta$ collapses the subspace $(*, t, y) \cup(x, t, *)$ to a point. Since the join is reduced, this subspace is contractible. Consequently $\alpha$ has a right homotopy inverse, and we can study $H(\sigma)$ by considering $H(\sigma) \alpha$ :

$$
H(\sigma)(\alpha(t, x, y))= \begin{cases}(x, 1-3 t), & 0 \leq t \leq 1 / 3 \\ (x / y, 3 t-1), & 1 / 3 \leq t \leq 2 / 3 \\ (* / y, 3-3 t), & 2 / 3 \leq t \leq 1\end{cases}
$$

Thus

$$
H(\sigma) \alpha=-S\left(\pi_{1}\right)+S\left(m\left(\pi_{1}, \gamma \pi_{2}\right)\right)-S\left(\gamma \pi_{2}\right)
$$

here we use associativity to write $* / y=\gamma(y)$ and $x / y=x \cdot \gamma(y)$. Now observe that

$$
(-S(\gamma)) \cdot H(\sigma) \alpha=S\left(\gamma \pi_{2}\right)-S\left(\gamma m\left(\pi_{1}, \gamma \pi_{2}\right)\right)+S \pi_{2} .
$$

Note that $\gamma m\left(\pi_{1}, \gamma \pi_{2}\right)=m\left(\pi_{2}, \gamma \pi_{1}\right)$, so

$$
\begin{aligned}
H(\sigma) \alpha(-S \tau) & =\left(-S\left(\pi_{2}\right)+S\left(m\left(\pi_{2}, \gamma \pi_{1}\right)\right)-S\left(\gamma \pi_{1}\right)\right)(-1) \\
& =S\left(\gamma \pi_{1}\right)-S m\left(\pi_{2}, \gamma \pi_{1}\right)+S\left(\gamma \pi_{2}\right),
\end{aligned}
$$

which completes the proof.

Finally we observe that in the case $p=2$ it is possible to have $R=T * T$. For an example let $T=R P^{\infty}$ and note that $G=S R P^{\infty}$ is atomic, even as a module over $\left\{S_{q}{ }^{1}, S_{q}{ }^{2}\right\}$. It follows that $R=R P^{\infty} * R P^{\infty}$. 
7.

In this section we will show that in many cases, an associative $H$ space structure on $T$ is not possible. The model for this is the case $G=P^{2 n+1}\left(p^{r}\right)$ of $\mathrm{CMN}$. In this case Neisenderfer has pointed out that the space $T_{0}^{2 n+1}$ which is a retract of $\Omega P^{2 n+1}\left(p^{r}\right)$ does not admit an associative $H$ space structure. The key fact here is that there is a class $v \in H_{2 n+1}\left(P^{2 n+1}\left(p^{r}\right)\right)$ with $\beta^{(r)}(v) \neq 0$. We will consider generalized Bockstein homology operations $\beta_{*}$ defined and natural on some full subcategory $\mathcal{C}_{\beta}$ of the category of $\mathrm{CW}$ complexes (for example, spaces on which $\beta^{(1)}, \ldots, \beta^{(r-1)}$ are all zero, and hence $\beta^{(r)}$ is a well-defined homology operation). We assume that $\mathcal{C}_{\beta}$ is closed under finite products colimits and retracts. Consequently it is closed under suspension and the James construction.

The operations we consider include both the Bockstein operations and the Milnor operations $Q^{i}$ as well as possible higher order Milnor operations. We assume a stable homology operation

$$
\beta: H_{i}(X) \longrightarrow H_{i-2 d-1}(X)
$$

defined and natural for $X \in \mathcal{C}_{\beta}$. We assume $\beta^{2}=0, \beta(\sigma(x))=\sigma(\beta(x))$, where $\sigma$ is the homology suspension, and

$$
\beta(x \times y)=\beta(x) \times y+(-1)^{|x|} x \times \beta(y) .
$$

Note that if $T \in \mathcal{C}_{\beta}, S T \in \mathcal{C}_{\beta}$ and hence $G \in \mathcal{C}_{\beta}$. Conversely, if $G \in \mathcal{C}_{\beta}$ and $G$ is homotopy associative and homotopy commutative, $\Omega G \in \mathcal{C}_{\beta}$ by Theorem 3.3. (Here we see the fact that $\beta$ is stable so $\beta$ can be defined on $X$ iff it can be defined on $S X$.) Consequently $T \in \mathcal{C}_{\beta}$.

Proposition 7.1. Suppose $G$ admits a homotopy co-associative and co-commutative structure, and $H_{*}(G)$ is $\beta$-acyclic. Suppose that $T$ admits a homotopy associative and commutative structure. Then $0=\beta: H_{2 n+1}(G) \longrightarrow H_{2 n-2 d}(G)$.

One may apply this result, for example, to the space

$$
V(1)^{n}=P^{n-2 p+1} \cup_{A} C P^{n-1}, \quad n \geq 2 p+4,
$$

where $A: P^{n-1} \longrightarrow P^{n-2 p+1}$ is the Adams map. We apply $Q_{0}$ in the case $n$ is odd and $Q_{1}$ in the case $n$ is even to see that the corresponding space $T\left(v_{1}\right)^{n-1}$ does not carry a homotopy associative and homotopy commutative $H$ space structure.

Proof. Suppose $v \in H_{2 n+1}(G)$ is such that $\beta v^{\prime} \neq 0$. Let $v=f_{*}\left(v^{\prime}\right) \in H_{2 n}(T)$ and $u=\beta v$. By Proposition 3.6, $u$ and $v$ are both primitive. Since $T$ is homotopy associative and commutative, $u^{2}=0$, while $u v^{i} \neq 0$ for $i<p$ by induction and applying $\Delta_{*}: H_{*}(T) \longrightarrow H_{*}(T) \otimes H_{*}(T)$. Also $\beta\left(u v^{p-1}\right)=-u^{2} v^{p-1}=0$. Since $H_{*}(G)$ is $\beta$-acyclic $H_{*}(\Omega G)$ and $H_{*}(T)$ are also $\beta$ acyclic. Consequently there is a class $w \in H_{2 n p}(T)$ with $\beta w=u v^{p-1}$. Define $\widetilde{\omega} \in H_{*}(T) \otimes H_{*}(T)$ by

$$
\widetilde{\omega}=\omega \otimes 1+\sum_{i=1}^{p-1} \frac{1}{p}\left(\begin{array}{l}
p \\
i
\end{array}\right) v^{i} \otimes v^{p-i}+1 \otimes \omega .
$$

Then

$$
\Delta_{*}\left(u v^{p-1}\right)=\sum_{i=1}^{p-1}\left(\begin{array}{c}
p-1 \\
i
\end{array}\right)\left\{u v^{i} \otimes v^{p-i-1}+v^{i} \otimes u v^{p-i-1}\right\}=\beta(\widetilde{\omega}) .
$$

Consequently $\beta\left(\Delta_{*}(\omega)-\widetilde{\omega}\right)=0$. 
Claim. If $z \in H_{*}(T) \otimes H_{*}(T)$ is a cycle it does not contain the term $v \otimes v^{p-1}$.

Proof. Write $z=\Sigma z_{i}$ with $z_{i} \in H_{i}(T) \otimes H_{m-i}(T)$. If $\beta z=0$, it follows that

$$
(\beta \otimes 1) z_{i}= \pm(1 \otimes \beta) z_{i-2 d-1}=0
$$

for all $i$ and hence $(\beta \otimes \beta) z_{i}=0$ for all $i$. Write $\langle x\rangle$ for the subspace spanned by $x$. Then

$$
\begin{aligned}
H_{2 n}(T) & =\langle v\rangle \oplus A, \\
H_{2 n-2 d-1}(T) & =\langle u\rangle \oplus B,
\end{aligned}
$$

and we can arrange $A$ and $B$ so that $\beta(a) \in B$ for each $a \in A$. Likewise write

$$
\begin{aligned}
H_{2 n(p-1)}(T) & =\left\langle v^{p-1}\right\rangle \oplus C, \\
H_{2 n(p-1)-2 d-1}(T) & =\left\langle u v^{p-2}\right\rangle \oplus D,
\end{aligned}
$$

with $\beta(c) \in D$ for all $c \in C$. Now

$$
\begin{aligned}
H_{2 n}(T) \otimes H_{2 n(p-1)}(T) \cong\left\langle v \otimes v^{p-1}\right\rangle \oplus\langle v\rangle \otimes C & \oplus A \otimes\left\langle v^{p-1}\right\rangle \\
& \oplus \quad A \otimes C .
\end{aligned}
$$

Applying $\beta \otimes \beta$ we obtain an element of

$$
\left\langle u \otimes u v^{p-2}\right\rangle \oplus\langle u\rangle \otimes D \oplus B \otimes\left\langle u v^{p-2}\right\rangle \oplus B \otimes D .
$$

Since $\beta \otimes \beta\left(v \otimes v^{p-1}\right)=\left\langle-u \otimes u v^{p-2}\right\rangle$, the term $v \otimes v^{p-1}$ is not present in any cycle. It is present in $\widetilde{\omega}$, so it must also be present in $\Delta_{*}(\omega)$. Since $T$ is primitively generated by Proposition 3.6, each term in $\omega$ is a sum of products of primitives; now

$$
\Delta_{*}\left(\omega_{1} \ldots \omega_{s}\right)=\sum c_{\alpha \beta} \omega_{\alpha} \otimes \omega_{\beta},
$$

where $c_{\alpha \beta}$ are coefficients and $\omega_{\alpha}, \omega_{\beta}$ are products of the $\omega_{i}$ in such a way that $\omega_{\alpha} \omega_{\beta}=\omega_{1} \ldots \omega_{s}$. Consequently if $\Delta_{*}(\omega)$ contains the term $v \otimes v^{p-1}$, it must come from a term $v^{p}$ in $\omega$. But $\Delta^{*}\left(v^{p}\right)=v^{p} \otimes 1+1 \otimes v^{p}$, so this is impossible.

\section{APPENDIX}

In this section we collect some general facts about Hopf map constructions. We thank Yukata Hemmi and Norio Iwase for some helpful e-mail conversations.

Proposition A1. There is a 1-1 correspondence between homotopy classes of maps $\theta: A \times F \longrightarrow F$ with $\theta(*, f)=f$ and fiber homotopy classes of fibrations

$$
F \longrightarrow E \longrightarrow S A,
$$

where $\theta$ is the restriction to $A \times F$ of the action map

$$
\Omega S A \times F \stackrel{a}{\longrightarrow} F
$$

defined by the homotopy lifting property.

Proof. For each such $\theta: A \times F \longrightarrow F$ we define a quasi-fibering

$$
E_{\theta}=F \cup_{\theta}(C A) \times F \longrightarrow S A,
$$

where the subspace $A \times F \subset(C A) \times F$ is identified with $F$ via $\theta$. Both the cone and the suspension are reduced. Thus construction is due to Dold and Lashof [DL], and it is shown in G2 that each Hurewicz fibering of the form considered here is 
homotopy equivalent to such a construction. Given a homotopy $\theta_{t}: A \times F \longrightarrow F$ with $\theta_{t}(*, f)=f$ we construct $\bar{\theta}: A \times(F \times I) \longrightarrow F \times I$ by $\bar{\theta}(a, f, t)=\left(\theta_{t}(a, f), t\right)$. From this we construct a quasi-fibering

$$
F \times I \longrightarrow E_{\bar{\theta}} \longrightarrow S A,
$$

and the inclusion of $E_{\theta_{0}}$ and $E_{\theta_{1}}$ into $E_{\bar{\theta}}$ are clearly homotopy equivalences.

To recover the map $\theta: A \times F \longrightarrow F$ from an arbitrary Hurewicz fibering we first discuss the action map $a: \Omega B \times F \longrightarrow F$ defined for each Hurewicz fibering

$$
F \longrightarrow E \longrightarrow B \text {. }
$$

This is constructed in a standard way by choosing a lifting in the diagram

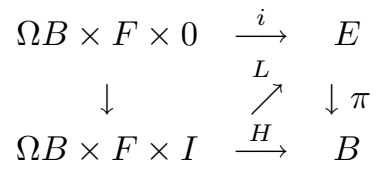

where $i(w, f, o)=f$ and $H(w, f, t)=w(t)$. Then $L(w, f, 1) \in F=\pi^{-1}(*)$, so by restriction $L$ defines the action map

$$
a=\left.L\right|_{\Omega B \times F \times 1}: \Omega B \times F \longrightarrow F .
$$

For each map $\psi: X \longrightarrow \Omega B$ we can, by restriction, construct a lifting $L^{\prime}$ in the diagram

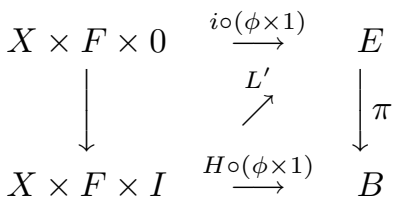

We now assert that given any two choices $L_{0}^{\prime}$ and $L_{1}^{\prime}$ of liftings, the associated "action maps"

$$
a_{0}, a_{1}: X \times F \longrightarrow F
$$

(defined by restricting to $X \times F \times 1$ ) are homotopic. To see this we define a map

$$
I: X \times F \times(I \times 0 \cup 0 \times I \cup 1 \times I) \cup * \times F \times I \times I \longrightarrow E
$$

by applying $L_{\epsilon}^{\prime}$ on $X \times F \times \epsilon \times I$ (for $\epsilon=0,1$ ) and projecting $X \times F \times I \times 0 \cup * \times F \times I \times I$ onto $F \subset E$. Define $J: X \times F \times I \times I \longrightarrow B$ by $J(x, f, s, t)=\psi(x)(t)$. We then extend $I$ to a homotopy covering $J$, and this homotopy, when restricted to $X \times F \times I \times 1$, is a homotopy between $a_{0}$ and $a_{1}$.

It follows that in the case of a fibering

$$
F \longrightarrow E \longrightarrow S A
$$

we have constructed a well-defined homotopy class of maps $\Omega S A \times F \stackrel{\theta^{\prime}}{\longrightarrow} F$ with $\theta^{\prime}(*, f)=f$. By restriction to $A \times F$ we obtain a class $\theta$. We only need to see that we can choose $\theta$ as a lifting for $E_{\theta}$ :

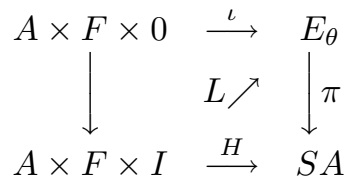

with $\iota(a, f)=f \in F \subset E_{\theta}, H(a, f, t)=(a, t) \in S A$ and $L(a, f, t)=((a, t), f) \in$ $C A \times F$. Here $C A=A \times I / A \times 0 \cup * \times I$ and $L(a, f, 1)=\theta(a, f) \in F \subset E$. 
Proposition A2. If the composition $A \times * \subset A \times F \stackrel{\theta}{\longrightarrow} F$ is a homotopy equivalence, $i: F \longrightarrow E_{\theta}$ is null homotopic and $E_{\theta} \simeq F * F$.

Proof. Since $\left.\theta\right|_{A \times *}$ is a homotopy equivalence, $i$ factors through $\left.\theta\right|_{A \times *}$ up to homotopy and hence it factors through $(C A) \times *$, so $i$ is null homotopic. $E_{\theta}$ is the reduced homotopy push-out of the diagram

$$
F \stackrel{\theta}{\longleftarrow} A \times F \stackrel{\pi_{2}}{\longrightarrow} F .
$$

We use the commutative diagram

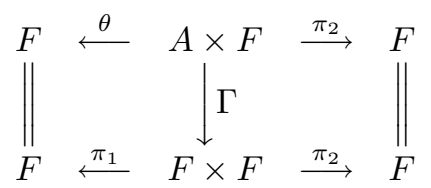

where $\Gamma(a, f)=(\theta(a, f), f)$. By hypothesis, $\Gamma$ is a homotopy equivalence, so $E_{\theta}$ is homotopy equivalent to the reduced homotopy push-out of

$$
F \stackrel{\pi_{1}}{\longleftarrow} F \times F \stackrel{\pi_{2}}{\longrightarrow} F
$$

which is the reduced join $F * F$.

Proposition A3. Replacing $\Omega S A$ by the James construction $A_{\infty}$, the action map

$$
a: A_{\infty} \times F \longrightarrow F
$$

is given by the formula

$$
a\left(\left(a_{1}, \ldots, a_{\alpha}\right), f\right)=\theta\left(a_{1}, \theta\left(a_{2}, \ldots, \theta\left(a_{k}, f\right) \ldots\right)\right) .
$$

Proof. One way to construct the action map is to replace the projection map by the canonical construction

$$
\begin{aligned}
& \bar{E}=\{(w, e) \in P B \times E \mid \pi(e)=w(0)\}, \\
& \bar{F}=\{(w, e) \in P B \times E \mid \pi(e)=w(0), w(1)=*\} .
\end{aligned}
$$

Then the action map $a: \Omega B \times \bar{F} \longrightarrow \bar{F}$ is given by

$$
a\left(w,\left(w^{\prime}, e\right)\right)=\left(w^{\prime \prime}, e\right),
$$

where

$$
w^{\prime \prime}(s)= \begin{cases}w^{\prime}(2 s), & 0 \leq s \leq \frac{1}{2} \\ w(2 s-1), & \frac{1}{2} \leq s \leq 1\end{cases}
$$

It is clear from this that the following diagram commutes up to homotopy:

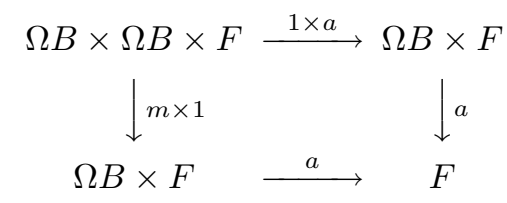

The result follows immediately since $A_{\infty}$ is generated by $A$ as a monoid. 


\section{REFERENCES}

[AG] D. Anick and B. Gray, Small H spaces related to Moore spaces, Topology 34, no. 4, (1995), 859-881. MR1362790 (97A:55011)

[AK] J.F. Adams and N.J. Kuhn, Atomic Spaces and Spectra, Proc. Edinborough Math. Soc. (2), 32, no. 3, (1989), 473-481. MR1015489 (90m:55010)

[B] I. Berstein, On co-groups in the category of graded algebras, Trans. Amer. Math. Soc. 115 (1965), 257-269. MR0206941(34:6757)

[BK] A.K. Bousfield and D.M. Kan, Homotopy limits, completions and localizations, LNM 304 (1972), Springer-Verlag. MR0365573 (51:1825)

[CM] F.R. Cohen, and M.E. Mahowald, A remark on the self maps of $\Omega^{2} S^{2 n+1}$, Indiana Univ. Math. J. 30 (1981), no. 4, 583-588. MR0620268 (82i:55013)

[CMN] F.R. Cohen, J.C. Moore, and J.A. Neisendorfer, Torsion in homotopy groups, Ann. of Math. (2) 109 (1979) no. 1, 121-168. MR0519355 (80e:55024)

[DL] A. Dold and R. Lashof, Principal quasifibrations and fiber homotopy equivalence of bundles, Illinois Journal of Mathematics 3, no. 2, (1959), 285-305. MR0101521 (21:331)

[F] P. Freyd, Stable Homotopy II, Proc. Symp. Pure Math XVII (1970), 161-183. MR0258028 $(41: 2675)$

[Ga] T. Ganea, Cogroups and Suspensions, Invent. Math. 9 (1970), 185-197. MR0267582 $(42: 2484)$

[G1] B. Gray, On Toda's fibrations, Math. Proc. Camb. Phil. Soc. 97 (1985), 289-298. MR0771822 (86i:55016)

[G2] B. Gray, On the Iterated Suspension, Topology 27, no. 3, (1988), 301-310. MR0963632 (89h:55016)

[G3] B. Gray, On the homotopy type of the loops on a 2-cell complex, Contemporary Mathematics, vol. 271 (2001), 77-98. MR1831348 (2002a:55010)

[G4] B. Gray, Unstable Families related to the image of J, Math. Proc. Comb. Phil. Soc. 96 (1984), 95-113. MR0743705 (86b:55014)

[H] J.R. Harper, Co-H-Maps to spheres, Israel Journal of Mathematics 66, nos. 1-3, (1989), 223-237. MR1017163(91b:55007)

[He] H.W. Henn, Finiteness properties of injective resolutions of certain unstable modules and applications, Math. Ann. 291 (1991), 191-203. MR1129359 (92i:55016)

[Hi] P.J. Hilton, On the homotopy groups of the union of spheres, J. London Math. Soc. 30 (1955), 154-172. MR0068218(16:847d)

[KSW] N.J. Kuhn, M. Slack, and F. Williams, Hopf constructions and higher projective planes for iterated loop spaces, TAMS 347 (1995) no. 4, 1201-1238. MR1282890 (95h:55008)

[M] J. Milnor, The Construction FK, Algebraic Topology: A Student's Guide, by J.F. Adams, Cambridge University Press, 1972.

[Ma] H. R. Margolis, Spectra and the Steemrod Algebra, North-Holland, 1983. MR0738973 (86j:55001)

[N] J. Neisendorfer, Product Decompositions of the double loops on odd primary Moore spaces, Topology 38, no. 6, (1999), 1293-1311. MR.1690159 (2000d:55024)

[S] M. Sugawara, On a condition that a space is an H-space, Math J. Okayama Univ. 6 (1957), 109-129. MR0086303 (19:160c)

[Se] P. Selick, A spectral sequence concerning the double suspension, Invent. Math. 64 (1981) no. 1, 15-24. MR0621768 (82j:55015)

[SW] P. Selick and J. Wu, On natural coalgebra decompositions of tensor algebras and loop suspensions, Memoirs AMS 148, no. 701 (2000). MR.1706247 (2001c:55006)

[T] S.D. Theriault, Homotopy decompositions involving the loops of coassociative Co-H spaces, Canad. J. Math. 55 (2003), no. 1, 181-203. MR.1952331 (2003k:55006)

[W] C. Wilkerson, Genus and Cancelation, Topology 14 (1975), 29-36. MR0367995 (51:4237)

Department of Mathematics, Statistics, and Computer Science (M/C 249), University of Illinois at Chicago, 851 South Morgan Street, Chicago, Illinois 60607-7045

E-mail address: brayton@uic.edu 